\title{
Frequency-Domain Optical Coherence Tomography Assessment of Human Carotid Atherosclerosis Using Saline Flush for Blood Clearance without Balloon Occlusion
}

\author{
C.A. Given II, G.F. Attizzani, M.R. Jones, C.N. Ramsey III, W.H. Brooks, M.A. Costa, and H.G. Bezerra
}

\begin{abstract}
SUMMARY FD-OCT is a new imaging technique that allows unprecedented in vivo microlevel assessment of human carotid plaque morphologic patterns and stent-vessel interactions. Prior reports describing the use of this technique have used balloon occlusion of the target vessel or iodinated contrast media to facilitate imaging. We report, for the first time, in vivo FD-OCT imaging of human carotid arteries without the use of iodinated contrast material or balloon occlusion techniques.
\end{abstract}

ABBREVIATIONS: $\quad F D=$ frequency-domain; $O C T=$ optical coherence tomography $; \mathrm{TD}=$ time-domain

$\mathbf{T}$ here is an increasing recognition of the importance of plaque morphologic features in carotid-related thromboembolic stroke. Inflammation leading to disruption of a thin-cap fibroatheroma and subsequent thrombus formation are features associated with so-called vulnerable or high-risk atherosclerotic plaques. Therefore, identification of such features has been increasingly recognized to be of utmost importance in stratifying stroke risk in the presence of carotid artery disease. ${ }^{1,2}$

Intravascular OCT, a relatively new light-based imaging technique that affords the highest axial resolution (10 $\mu \mathrm{m})$ among any currently available intravascular imaging technique, is approved for coronary use in the United States. ${ }^{3}$ In particular, OCT has proved invaluable in identification of "high-risk" features in coronary plaques. ${ }^{4-9}$ Although OCT imaging of carotid artery plaque is currently in its infancy, previous case series suggest that the pathologic features of "vulnerable" plaques validated in the coronary arteries are similar to those seen in the carotid vasculature. $^{10-13}$

Although a detailed technical description of OCT performance is beyond the scope of this case series, it should be noted that the earliest published descriptions of carotid OCT imaging used TD-OCT, ${ }^{11-14}$ an early iteration of OCT that requires proximal balloon occlusion to effectively "clear" light-attenuating red

Received July 30, 2012; accepted after revision October 2

From the Central Baptist Hospital (C.A.G. II, M.R.J., C.N.R. III, W.H.B.), Baptist Heart and Vascular Institute, Lexington, Kentucky; and Harrington Heart and Vascular Institute (G.F.A., M.A.C., H.G.B.), University Hospitals, Case Medical Center, Case Western Reserve University, Cleveland, Ohio.

Please address correspondence to Curtis A. Given II, MD, 1720 Nicholasville Rd, \#601, Lexington, KY 40503; e-mail: cgiven2@insightbb.com

--m Indicates open access to non-subscribers at www.ajnr.org

http://dx.doi.org/10.3174/ajnr.A3411 blood cells during image acquisition. The newest iteration of OCT_FD-OCT - allows very rapid data acquisition (up to 25 $\mathrm{mm} / \mathrm{s}$ ), thus obviating the need for balloon occlusion, with image acquisition carried out during iodinated contrast injection. Our group and other researchers recently reported initial experiences with FD-OCT imaging without balloon occlusion in the carotid vasculature. ${ }^{10,15}$ Nevertheless, although FD-OCT solves the hurdle of balloon occlusion posed by TD-OCT, contrast injection for clearance of red blood cells is still a potential concern, particularly in patients at higher risk for contrastinduced nephropathy. ${ }^{10,13}$

We report, for the first time, the successful use of FD-OCT in vivo to evaluate carotid artery disease by using only saline flush to clear red blood cells during image acquisition. This latest variation of FD-OCT imaging allows for image acquisition without the need for balloon occlusion or iodinated contrast material.

\section{Patient Series}

From November 2011 to May 2012, we performed ad hoc carotid artery FD-OCT examinations using heparinized saline for blood pool clearance in 10 patients. All studies were performed at the Baptist Heart and Vascular Institute with FD-OCT used for clinical management purposes in the reported patients, and retrospective review and reporting of cases were approved by the Institutional Review Board. The FD-OCT examinations were performed to clarify ambiguous DSA findings and/or discordant noninvasive data (8 patients), or as part of the evaluation for a presumed thromboembolic source in the setting of an acute cerebrovascular accident with documented diffusion abnormality on MR imaging (2 patients). Six of the patients were men, and the patients ranged in age from $58-76$ years (mean age, 70 years). Six patients were symptomatic, defined as having a documented 


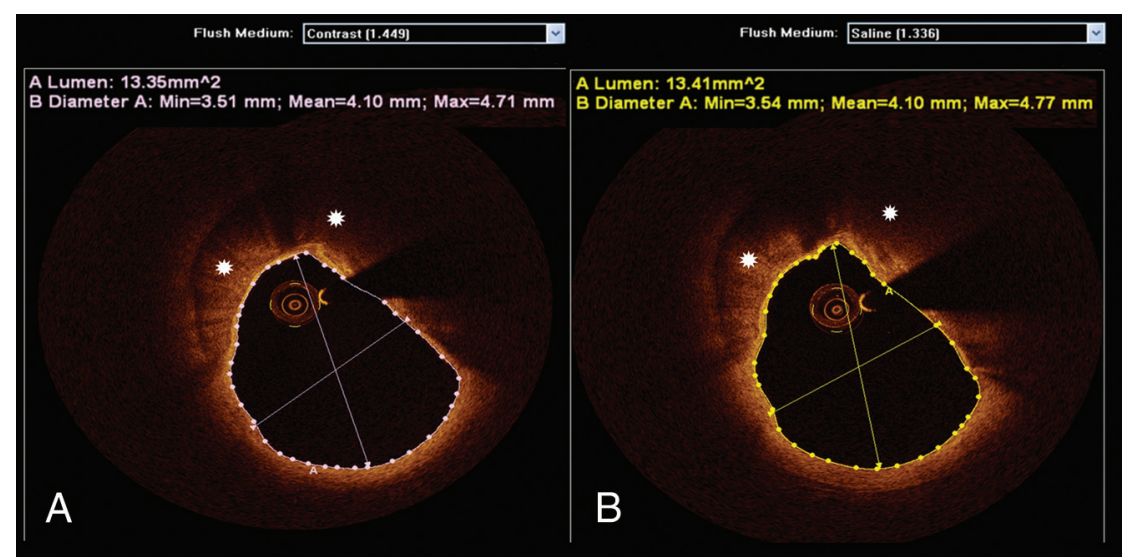

FIG 1. FD-OCT quantitative assessments with contrast and saline injections. An FD-OCT cross-sectional image of the ICA obtained after undiluted iodinated contrast injection is depicted in $(A)$. Luminal measurements and calcified plaque (asterisks) are demonstrated. The same artery (coregistered) was imaged by FD-OCT after heparinized saline injection ( $B$ ), illustrating comparable luminal dimensions and plaque morphologic patterns. Note that the dedicated software used for analysis enables the selection of different flush media to account for different index of refraction.

stroke or transient ischemic attack within 90 days before the FDOCT procedure. Seven patients had FD-OCT interrogations with separate injections of iodinated contrast followed by heparinized saline to clear red blood cells. FD-OCT imaging was performed with only saline flush in 3 patients because of concerns regarding renal function. No adverse effects were associated with FD-OCT imaging or DSA studies.

The examinations were performed via transfemoral approach by use of either $6 \mathrm{~F}$ or $7 \mathrm{~F}$ long sheaths placed into the suspect carotid artery, in a fashion similar to that previously reported. ${ }^{10}$ All patients were given a heparin bolus $(50-100 \mathrm{U} / \mathrm{kg})$ to reach a target activated clotting time of more than 250 seconds. The $2.7 \mathrm{~F}$ C7 Dragonfly FD-OCT catheter (St. Jude Medical, St Paul, Minnesota) was advanced across the ICA origin under fluoroscopic guidance. In 7 patients, the FD-OCT catheter was advanced across a 0.014-inch microguidewire (Transcend EX soft tip; Target Therapeutics, Fremont, California). In the remaining 3 patients, the FD-OCT catheter was navigated over a previously deployed filterwire distal embolic protection device (NAV-6; Abbott Vascular, Redwood City, California). We acquired data by using a commercially available FD-OCT system (C7-XR, OCT Imaging System; St. Jude Medical). The images were calibrated by automated adjustment of the Z-offset, and automated pullback was set at 20 $\mathrm{mm} / \mathrm{s}$. During imaging acquisition, blood was displaced by injection of undiluted iodinated contrast. This contrast consisted of a hand injection of $25 \mathrm{~mL}$ of Visipaque 320 (GE Healthcare, Piscataway, New Jersey) for a 5 -second period $(5 \mathrm{~mL} / \mathrm{s})$ via a $30-\mathrm{mL}$ syringe or heparinized $(2 \mathrm{U} / \mathrm{mL}$ ) saline solution (hand injection of $50 \mathrm{~mL}$ for an approximate 5 -second period $[10 \mathrm{~mL} / \mathrm{s}]$ via a 60 -mL syringe) through the long sheath. $2 \mathrm{D}$ images were reconstructed at the time of acquisition and were used for clinical decision-making purposes while the patient was in the angiography suite. An independent core laboratory (Cardiovascular Imaging Core Laboratory, Harrington Heart and Vascular Institute, University Hospitals, Case Medical Center, Cleveland, Ohio) reviewed all FD-OCT images. The images were analyzed by 2 independent investigators (G.F.A., H.G.B.) blinded to the angiographic and clinical data and were reviewed by a third reader
(M.A.C.). We performed FD-OCT analyses by using dedicated software with an automated contour-detection algorithm (Offline Review Software, version C.0.2; St Jude Medical).

All cross-sectional images (frames) of the ICA were initially screened for quality assessment and were considered not suitable for analysis if any portion of the image was out of the screen, presenting foldover artifacts, ${ }^{3}$ or if the presence of intraluminal blood impaired the assessment of a continuous arc of at least $270^{\circ} .^{16}$ Qualitative image assessment was performed in every frame (ie, every $0.2 \mathrm{~mm}$ ), whereas quantitative measurements of lumen areas and diameters in native carotid arteries were performed in every 5 frames (ie, every $1 \mathrm{~mm}$ ). Comparisons between luminal dimensions obtained with saline and contrast injections were performed in coregistered lengths of the ICA with use of the common carotid bifurcation as a landmark. Although the refractive indices of saline (approximately 1.336) and contrast (approximately 1.449) are different, the proprietary software used for analysis (Off-line Review Software, version C.0) allows automated adjustments of the refractive index, enabling accurate assessments regardless of flush media used (Fig 1).

One thousand two hundred twenty-eight frames, and 1022 frames were analyzed with saline and contrast injections, respectively. Saline injections were able to achieve analyzable images in $100 \%$ of the frames, whereas contrast injection obtained analyzable images in $95.3 \%$ of the frames. Among the images not suitable for analysis that were obtained with contrast injection, $100 \%$ exhibited residual blood, $60.2 \%$ were out of screen, and $21 \%$ had foldover artifacts. No discrepancy between saline and contrast regarding quantitative luminal measurements or qualitative plaque assessment was revealed in coregistered frames (Figs 1-3). Quantitative luminal assessment was performed on 221 frames in the saline and contrast groups, enabling $t$ test analysis for differences between contrast and saline luminal measurements. There was no significant difference between the 2 methods in mean luminal area (mean difference, $-0.355 ; t$ value, $-1.0808 ; P=.168$ ) and in mean luminal diameter (mean difference, -0.050 ; $t$ value, -1.496; $P=.232$ ). 

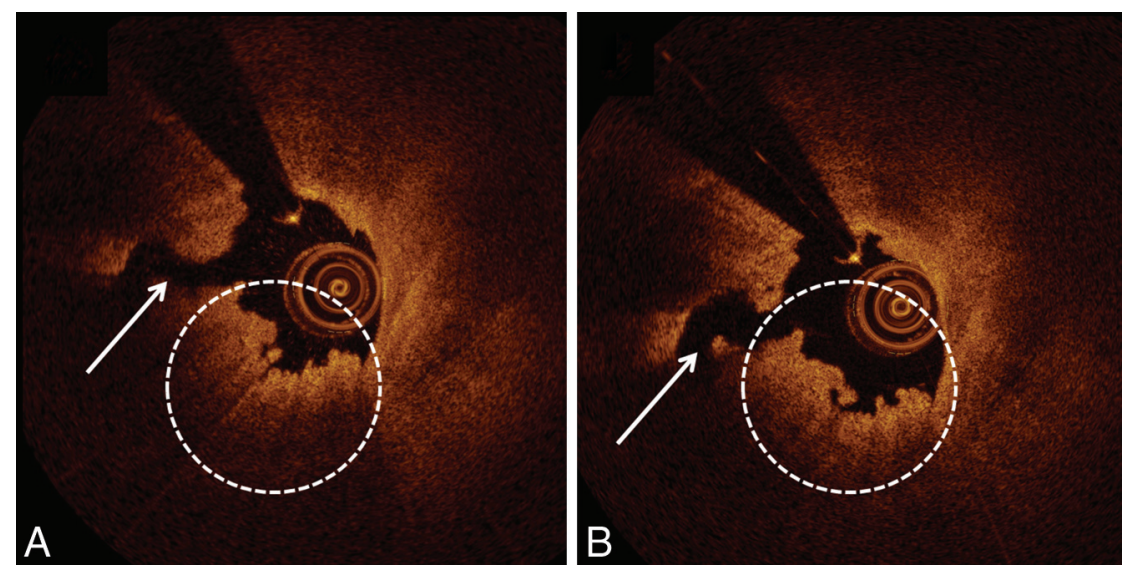

FIG 2. FD-OCT qualitative assessments with contrast and saline injections. FD-OCT cross-section of the ICA was obtained with contrast injection $(A)$. A ruptured plaque (white arrow) with overlying red thrombus (white dashed circle) is depicted. The same artery imaged by FD-OCT after saline injection (B) demonstrates similar features.
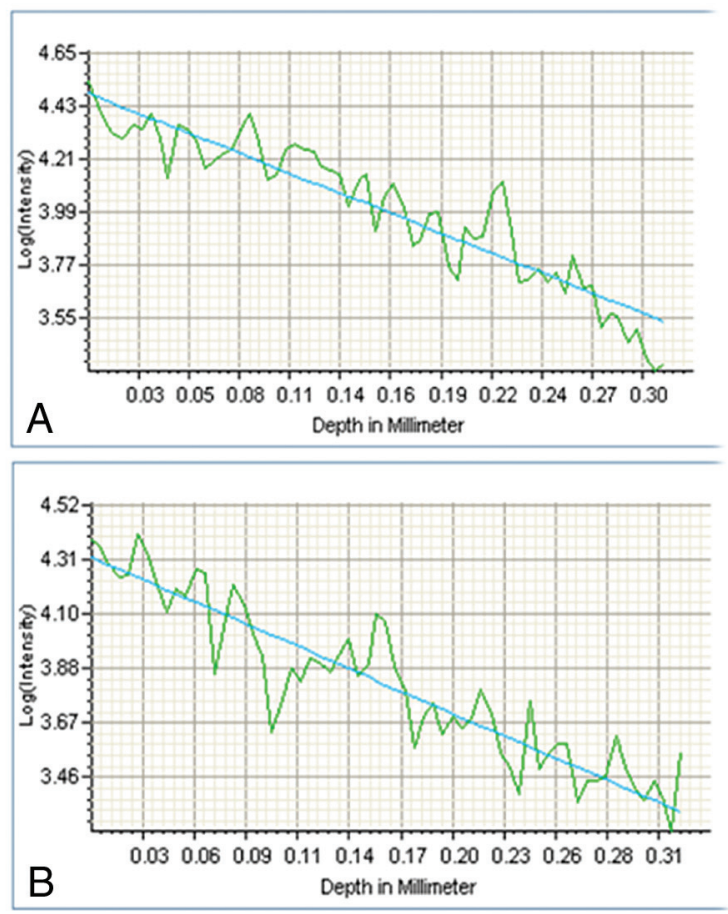

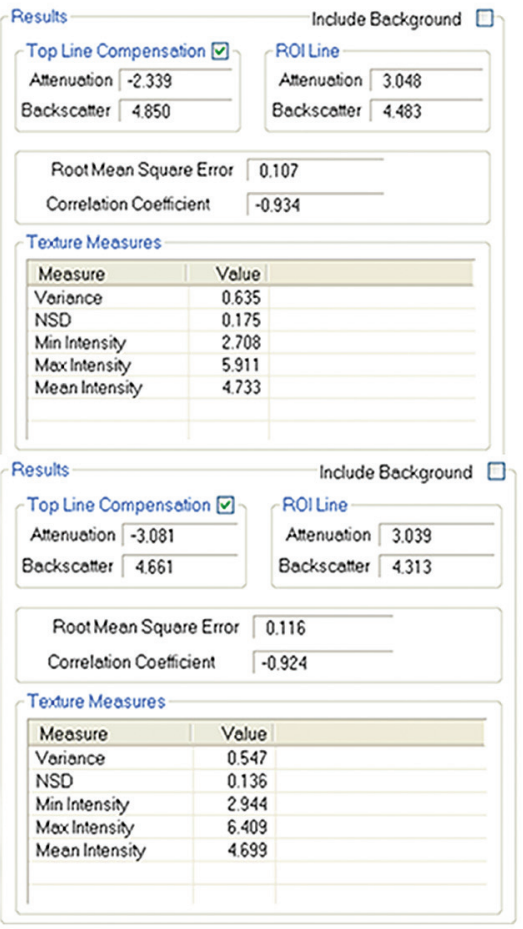

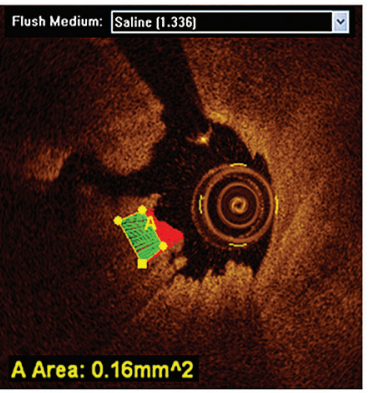

FIG 3. Red thrombus optical properties obtained by means of FD-OCT after contrast and saline injections. FD-OCT assessment of ICA thrombus after contrast injection $(A)$ includes a graphic representation of sharply attenuated light consistent with red thrombus. In (B), the same coregistered segment of artery was imaged by FD-OCT after saline injection, with demonstration of comparable optical properties.

\section{DISCUSSION}

Red blood cells attenuate light. Therefore, they must be effectively displaced from the vessel lumen to permit OCT imaging. Saline has been shown to be an effective agent for red blood cell clearance during TD-OCT studies done with balloon occlusion in the coronary and carotid arteries. ${ }^{11,12,17,18}$ Although FD-OCT, the newest iteration of OCT, eliminates the need for balloon occlusion with its rapid acquisition rate, blood cell clearance by use of this technique has historically required a more viscous agent (eg, iodinated contrast material) to provide effective clearance of red blood cells during periods of uninterrupted blood flow. ${ }^{3,19}$ Previous studies of carotid OCT imaging have reported using either iodinated contrast material with FD-OCT or saline and balloon occlusion with TD-OCT to achieve satisfactory blood clearance for "diagnostic" quality images. ${ }^{10-14}$ We hypothesized that the carotid vasculature would accommodate significantly higher volumes of flush media compared with the coronary arteries, allowing for effective blood clearance with saline injections during FDOCT imaging. Although heparinized saline required larger volumes at higher injection rates (compared with the iodinated contrast material), these injection rates were within acceptable ranges for carotid angiography. ${ }^{20,21}$ Red blood cell clearance with saline flush was at least as effective as iodinated contrast material in our patients (100\% vs $95.3 \%$ ) for coregistered frames, and was successful even with "large" internal carotid arteries (Figs 4 and 5). 


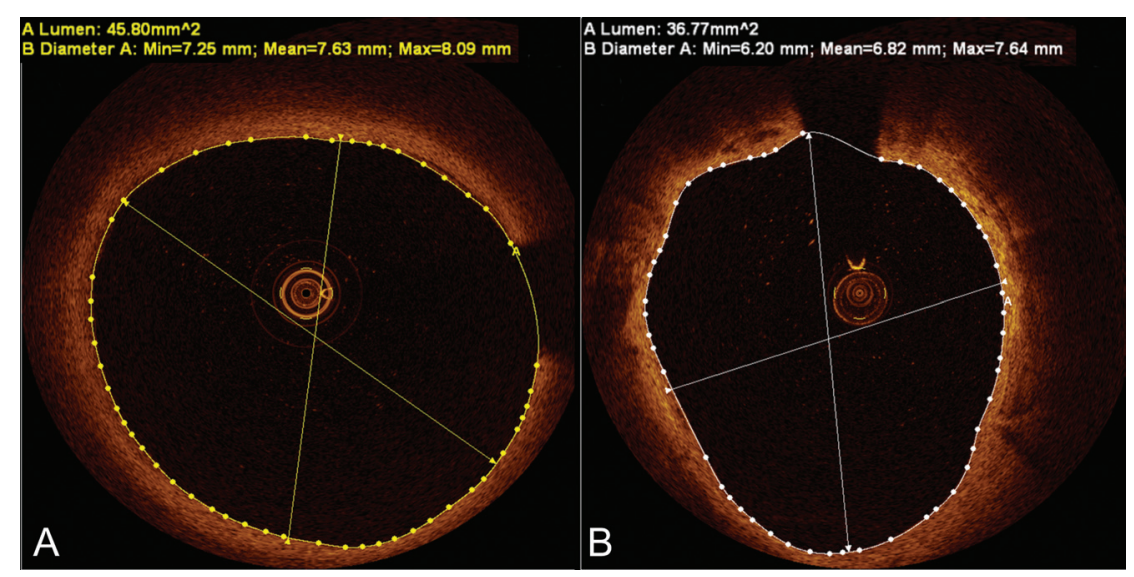

FIG 4. Satisfactory blood clearance with saline injections. Frequency-domain optical coherence tomography cross-sections of large internal carotid arteries in $(A)$ and $(B)$ demonstrate excellent blood clearance with saline injections, despite large lumen dimensions.
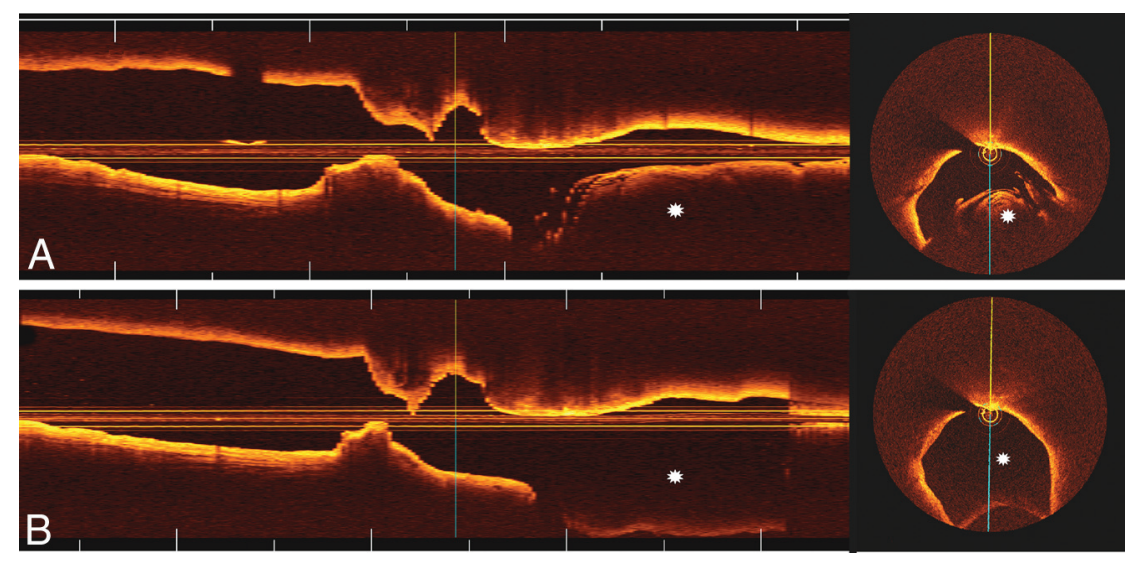

FIG 5. FD-OCT images with contrast and saline injections. Longitudinal and cross-sectional FD-OCT images of the ICA obtained with contrast $(A)$ and saline $(B)$ flush media, demonstrate comparable blood clearance at the target lesion (area of luminal narrowing) within the ICA. In this particular patient, improved blood clearance with saline flush (compared with contrast) was observed in the more proximal common carotid artery (asterisks).

Dextran has recently been shown as a viable substitute for contrast material in coronary FD-OCT imaging. ${ }^{22,23}$ Although the usefulness of dextran in carotid FD-OCT imaging is unknown, we suspect that it would provide similar diagnostic quality images with adequate clearance of red blood cells. However, dextran has been associated with nephrotoxicity and anaphylactoid reactions. ${ }^{24,25}$ Thus, we believe that heparinized saline solution promises to be a superior agent for carotid OCT imaging. In rare instances of known heparin allergy, image acquisition could likely be achieved in a similar fashion with nonheparinized saline flush. Another factor to consider is the higher cost of dextran solutions relative to heparinized saline.

The role that OCT will play in the evaluation of carotid plaque morphologic patterns remains speculative at this time. Because of the "noninvasive" nature of duplex ultrasonography, CT, and MR imaging, these techniques would most likely be used in future clinical-related evaluations of carotid plaque histopathologic features. However, carotid OCT offers promise both as a research tool for the in vivo validation of these techniques and as a clinical tool in the evaluation of unexpected ambiguous findings during DSA and stent-vessel interactions after stent placement, as this anatomic situation adversely affects MR and CT imaging.
Our group was the first to demonstrate the technical feasibility of FD-OCT without balloon occlusion for the assessment of carotid artery disease. ${ }^{10}$ Setacci et $\mathrm{al}^{15}$ have subsequently confirmed the safety and feasibility of performing FD-OCT assessment in the carotid vasculature, reporting similar percentages of technical success (97.3\%) using contrast material for blood clearance. Our present series extends these initial findings to validate the feasibility of data acquisition without use of contrast material. Moreover, elimination of the previously reported obligatory $25-\mathrm{mL}$ contrast per acquisition was achieved without compromise in image quality. The acquisition of images with unprecedented resolution without special accommodations or the need for contrast material makes FD-OCT a promising tool for the assessment of carotid artery disease and carotid stent placement.

Disclosures: Guilherme Attizzani-UNRELATED: Consultancy: St. Jude Medical. Michael Jones-UNRELATED: Payment for Lectures (including service on speaker bureaus): St. Jude Medical, Comments: speaker bureau. Marco Costa—UNRELATED: Consultancy: St. Jude Medical; Grants/Grants Pending: St. Jude Medical; Payment for Lectures (including service on speaker bureaus): Abbott, Medtronic, Boston Scientific, Cordis; Patents (planned, pending, or issued): patent of University Hospitals for OCT software. *Hiram Bezerra-UNRELATED: Consultancy: St. Jude Medical; Payment for Lectures (including service on speaker bureaus): St. Jude Medical. *Money paid to institution. 


\section{REFERENCES}

1. Freilinger TM, Schindler A, Schmidt C, et al. Prevalence of nonstenosing, complicated atherosclerotic plaques in cryptogenic stroke. JACC Cardiovasc Imaging 2012;5:397-405

2. Lindsay AC, Biasiolli L, Lee JM, et al. Plaque features associated with increased cerebral infarction after minor stroke and TIA: a prospective, case-control, 3-T carotid artery MR imaging study. JACC Cardiovasc Imaging 2012;5:388-96

3. Bezerra HG, Costa MA, Guagliumi G, et al. Intracoronary optical coherence tomography: a comprehensive review clinical and research applications. JACC Cardiovasc Interv 2009;2:1035-46

4. Kume T, Akasaka T, Kawamoto T, et al. Assessment of coronary arterial plaque by optical coherence tomography. Am J Cardiol 2006;97:1172-75

5. Kume T, Okura H, Kawamoto T, et al. Assessment of the coronary calcification by optical coherence tomography. EuroIntervention 2011;6:768-72

6. Kubo T, Imanishi T, Takarada S, et al. Assessment of culprit lesion morphology in acute myocardial infarction: ability of optical coherence tomography compared with intravascular ultrasound and coronary angioscopy. J Am Coll Cardiol 2007;50:933-39

7. Yabushita H, Bouma BE, Houser SL, et al. Characterization of human atherosclerosis by optical coherence tomography. Circulation 2002;106:1640-45

8. Stamper D, Weissman NJ, Brezinski M. Plaque characterization with optical coherence tomography. J Am Coll Cardiol 2006;47: C69-79

9. Bezerra HG, Attizzani GF, Costa MA. Three-dimensional imaging of fibrous cap by frequency-domain optical coherence tomography. Catheter Cardiovasc Interv 2011 Sep 27 [Epub ahead of print].

10. Jones MR, Attizzani GF, Given CA 2nd, et al. Intravascular frequency-domain optical coherence tomography assessment of atherosclerosis and stent-vessel interactions in human carotid arteries. AJNR Am J Neuroradiol 2012;33:1494-501

11. Yoshimura S, Kawasaki M, Yamada K, et al. Visualization of internal carotid artery atherosclerotic plaques in symptomatic and asymptomatic patients: a comparison of optical coherence tomography and intravascular ultrsound. AJNR Am J Neuroradiol 2012;33: $308-13$

12. Yoshimura S, Kawasaki M, Hattori A, et al. Demonstration of intraluminal thrombus in the carotid artery by optical coherence tomography: technical case report. Neurosurgery 2010 Sep;67(3 Suppl Operative):onsE305; discussion onsE305

13. Reimers B, Nikas D, Stabile E, et al. Preliminary experience with optical coherence tomography imaging to evaluate carotid artery stents: safety, feasibility and techniques. EuroIntervention 2011;7: $98-105$

14. Yoshimura S, Kawasaki M, Yamada K, et al. OCT of human carotid arterial plaques. JACC Cardiovasc Imaging 2011;4:432-36

15. Setacci C, de Donato G, Setacci F, et al. Safety and feasibility of intravascular optical coherence tomography using a nonocclusive technique to evaluate carotid plaques before and after stent deployment. J Endovasc Ther 2012;19:303-11

16. Takarada S, Imanishi T, Liu Y, et al. Advantage of next-generation frequency-domain optical coherence tomography compared with conventional time-domain system in the assessment of coronary lesion. Catheter Cardiovasc Interv 2010;75:202-06

17. Guagliumi G, Bezerra HG, Sirbu V, et al. Serial assessment of coronary artery response to paclitaxel-eluting stents using optical coherence tomography. Circ Cardiovasc Interv 2012;5:30-38

18. Guagliumi G, Ikejima I, Sirbu V, et al. Impact of drug release kinetics on vascular response to different zotarolimus-eluting stents implanted in patients with long coronary stenoses: the LongOCT study (Optical Coherence Tomography in Long Lesions). J Am Coll Cardiol Intv 2011;4:778-85

19. Li X, Villard JW, Ouyang Y, et al. Safety and efficacy of frequency domain optical coherence tomography in pigs. EuroIntervention 2011;7:497-504

20. Yousem DM, Trinh BC. Injection rates for neuroangiography: results of a survey. AJNR Am J Neuroradiol 2001;22:1838-40

21. Morris PP, ed. Performing a cerebral or spinal arteriogram. In Practical Neuroangiography. Baltimore: Lippincott Williams \& Wilkins; $1997: 48$

22. Ozaki Y, Kitabata H, Tsujioka H, et al. Comparison of contrast media and low-molecular-weight dextran for frequency-domain optical coherence tomography. Circ J 2012;76:922-27

23. Brezinski M, Saunders K, Jesser C, et al. Index matching to improve optical coherence tomography imaging through blood. Circulation 2001;103:1999-2003

24. Data JL, Nies AS. Drugs five years later: dextran 40. Ann Intern Med 1974;81:500-04

25. Seeliger E, Flemming B, Wronski T, et al. Viscosity of contrast media perturbs renal hemodynamics. J Am Soc Nephrol 2007;18:2912-20 